\title{
Uses of Personalized Medicine in Current Pediatrics
}

\author{
Taha I. Yousif ${ }^{\mathrm{a}, \mathrm{c}}$, Khaled Bizantia ${ }^{\mathrm{a}}$, Basil Elnazir ${ }^{\mathrm{b}}$
}

\begin{abstract}
The development of personalized medicine (PM) has shifted medicine from "one size fits all" principle to tailoring treatment to patients' characteristics to get the intended benefits in a faster time with the least possible harm. In the early days whenever a patient is diagnosed with a certain disease, a known first-line medicine will be prescribed. If this first line fails, then we would follow the algorithm and use the next one. In the last century with the wide use of researches and massive volume of publications, the concept of evidence-based medicine has made a huge change in modern medicine. It is expected that the new millennium will be the PM era. PM in its simplest understanding is providing a tailored medication according to the patient's need. After using appropriate tool to identify the patient's genetic characteristics, a specific treatment with minimal side effects and higher rate of response would be prescribed. The first uses of this concept emerge from adult practice and the use of a specific treatment for patients with specific enzyme defects in cystic fibrosis, as we will discuss in details below. Oncology is the area that currently uses PM more than any other medical speciality. The aim of this article was to describe what the PM is about and what could be its expected benefits. We have reviewed the literature and obtained the most relevant articles. We will then mention some examples of applying PM in current pediatrics.
\end{abstract}

Keywords: Personalized; Personalized medicine

\section{Introduction: What Is Personalized Medicine (PM)?}

It is far more important to know what person the disease has than what disease the person has, said Hippocrates.

The simplest definition of PM comes from the Pharma-

\footnotetext{
Manuscript accepted for publication March 17, 2016

${ }^{a}$ Gastroenterology Department, Great Ormond Street Hospital, WC1N 1JH, London, UK

${ }^{b}$ Respiratory Paediatrics Department, Tallaght Hospital, Dublin 16, Dublin, Ireland

${ }^{\mathrm{c} C}$ Corresponding Author: Taha I. Yousif, Gastroenterology Department, Great Ormond Street Hospital, WC1N 1JH, London, UK.

Email: drtahaibrahim@gmail.com
}

doi: http://dx.doi.org/10.14740/ijcp241w ceutical Committee of European Commission "The provision of the right treatment to the right patient at the right dose at the right time" [1]. PM is not a new concept. Since the emergence of modern medicine, all the patients with a certain disease or diagnosis get the same medicine. As our knowledge broadens, we discovered that not all medicines work in the same way for all patients and moreover their effects are more prominent in others. An example of PM is the patients with glucose 6 phosphate dehydrogenase deficiency (G6PD), which is a red cell enzyme. Those patients were noticed to develop hemolytic reaction with many medications. These include quinolones such as primaquine, an anti-malarial. Other examples include non-steroidal anti-inflammatory medications, tylenols, and many other medications. This discovery has changed medical practice a lot. Patients with malaria nowadays should ideally be tested for G6PD deficiency before starting primaquine, if it is intended to be used. If they test negative, then they will be started on primaquine; otherwise it will be changed to a different medicine. This is an example of PM, tailoring the medicine according to the patient biologic, genetic and genomic characteristics.

\section{Definition of PM}

Several definitions exist to describe PM. These definitions range in spectrum from a really tight one to a much broader concept: personalized medicine coalition (PMC) [2], for example, defines PM as "The use of new methods of molecular analysis for a better management of a patient's disease or predisposition to disease".

The American Medical Association on the other hand defines PM as "health care that is informed by each person's unique clinical, genetic, and environmental information" [3]. Another definition comes from the National Cancer Institute to include prevention and diagnosis. It declares that PM is " $\mathrm{A}$ form of medicine that uses information about a person's genes, proteins, and environment to prevent, diagnose, and treat disease" [4].

\section{Requirement of PM}

PM involves the use of two tools, diagnostic and therapeutic. The diagnostic tool is used to stratify the disease. It may inform us about the patient's characteristic pharmacogenetics which may lead us to use specific medicine when or even be- 
fore the suspected disease is confirmed.

\section{Benefits of PM}

PM can [5]: 1) shift the emphasis in medicine from treatment to prevention: this would be achievable if we can anticipate which individual will get this disease, the thing which will help patients to get benefit from early preventive treatment; 2) guide the correct selection of treatment: this could be possible by avoiding the trial and error prescribing which leads to delay in initiating the correct treatment; this will eventually lead to high levels of compliance with treatment; 3) by achieving the above goals and preventing treatment delays, quality of life of patients will definitely improve which is one of the ultimate goals of treatment; 4) providing cost-effective treatment.

\section{Biomarkers and PM}

For many adult diseases and a few childhood diseases, some biomarkers were discovered to describe the inter-personal variations. These biomarkers exist as genes, proteins, and mRNA and could predict not only occurrence of a disease but also severity and response to various available treatments. Various human leucocyte antigens (HLAs) were linked to different reactions. HLA-B*1502 for example has been linked to severe adverse drug reaction with carbamazepine. Use of carbamazepine in patients who test positive to this biomarker may lead to severe skin necrolysis and Steven-Johnson syndrome [6, 7]. HLA-B 5701 similarly has been linked to hypersensitivity reaction to abacavir, an HIV drug $[6,8,9]$. In these examples, screening for theses HLA types will avoid giving the relevant drug to those patients who are at risk of developing adverse reaction and also gaining time on starting the right treatment at the right time. According to Scarpa et al, "In future, it is expected that such biomarkers will be detected more readily thanks to the new high-throughput technologies and powerful analytical approaches employed within the fields of pharmacogenomics, proteomics, metabolomics and other so-called "omics" [6].

Adverse reaction to medications (ADRs) accounts for 5\% of all hospital admissions. Various genes affect the processing and handling of these drugs resulting in these reactions. Cytochrome P450 CYP450 is one example $[2,10]$. Some people are fast metabolizers, and hence they utilize the drug quickly. Others are slow metabolizers with difficulties in degrading down some drugs, leading to high risk of intoxication and ADRs. Several tests are available to detect variations in CYP450 genes.

Warfarin, an anticoagulant that is widely used, gives us another good case to prove the value of PM. It has been noted that the required dose is subject to trial and error that could result in increased risk of bleeding or thrombosis. Recently, it was discovered that warfarin metabolism is affected by genetic variations. It requires an enzyme CYP2C9 to activate vitamin $\mathrm{K}$. The FDA now requires genotyping for patients prior to use of warfarin $[2,11]$. Despite identification of many biomarkers in research settings, unfortunately only few biomarkers are currently in clinical use in pediatrics. This is mainly due to issues with validation, which will require linking the existing networks together in order to widen the samples stored and share important data. This is expected to face many obstacles specially getting enough funding.

Currently many networks have been created to help gathering, and distributing and coordinating data about genetics related to drugs and the patients' response to treatments. One of the best examples of these is the Canadian pharmacogenomics network for drug safety (CPND). CPND is very active in collecting detailed information on ADRs and operates within Canadian teaching hospitals. It is playing a great role, not only in reducing ADRs by identifying genetic markers that could help pick up those patients at an increased risk of developing ADRs, but also in characterizing patients who may have less response to treatment [12]. This has helped in creating a huge dataset from genomic positions and bio-banks, which lead to the development of genome wide association studies (GWASs). GWASs look at nucleotide polymorphism across the genome, which makes it a huge step in understanding common diseases.

\section{PM and Pediatric Medicine}

Pharmacogenetic studies in pediatric medicine become more popular over the last few years especially in respiratory, oncology, gastroenterology and a lot of potentials in neurology fields. Here we will describe few examples of uses of PM in current pediatrics.

\section{Pediatric respiratory diseases and PM}

Respiratory medicine was one of the earliest specialities to benefit from PM. Using genotypes to guide treatment has helped in reducing side effects and time to reach the best available treatment. Ivacaftor was the first mutation-targeted therapy in cystic fibrosis patients. It potentiates cystic fibrosis transmembrane regulator (CFTR) protein class III mutations [13] by opening the channels and improves fluid regulation within affected cells. Studies in patients with a G551D mutation, which is the most common class III mutation, showed significant benefits. Two RCTs in children with G551D-CFTR mutation (ENVSION [14] and STRIVE Studies [15]) over 48 weeks period showed great benefits of this new medicine [16]. Symptoms improved a lot as well as spirometry and number of episodes exacerbation. There was also a reduction in sweat chloride. All these facts demonstrated that this treatment is life changing to patients with CF. This provides an excellent example of a stratified approach, which benefits patients who have diseases with different phenotypes. This has opened the door wide for future researches which could help identify other enzymes defects with other phenotypes of $\mathrm{CF}$ and developing the relevant medications. Based on the success of ivacaftor, a new medicine is being developed, VX-lumacaftor [17]. Re- 
cent studies concluded that lumacaftor in combination with ivacaftor provided a benefit for patients with cystic fibrosis homozygous for the Phe508del CFTR mutation [16]. Very recently, in July 2015, the FDA approved a new drug ORK$\mathrm{AMBI}^{\mathrm{TM}}$ (lumacaftor/ivacaftor) for treatment of patients with homozygous F508del mutation 12 years of age or older. The F508del is the most common CFTR mutation, with approximately $45 \%$ of patients with CF being homozygous for this allele [16]. The cost is a real issue as currently it is 250,000 - 290,000 dollars per patient per year.

In asthma, which is a very common childhood disease, multiple GWASs were published. These were mainly describing development of asthma or susceptibility to chronic obstructive pulmonary diseases. IL-33 has been described as an important molecule in airway modeling. This could lead to future development of asthma cure, although it is still too early to talk about complete cure [18]. Basu et al in a study published in Journal of Allergy and Clinical Immunology [19] showed that patients with a certain beta-2 adrenergic receptor gene, carried by one in seven patients, are usually unresponsive to the salmeterol. Salmeterol is a long acting beta-2 agonist that is used as a second line of asthma treatment. Instead the study showed beneficial effects from use of montelukast and suggested its use rather than salmeterol in patients with this variant. Although the sample was small (62 subjects), it had a huge impact on clinical practice. As patients with this variant could be identified by a sputum test costing only $£ 15$, it would be a very cost-effective way to identify those patients and treat them promptly. Another study showed that a novel pharmacogenomic rs37973 has decremental effect on the expression of GLCCI1, a gene influencing the pharmacologic response to inhaled glucocorticoids in asthma [20]. Hence patients with this novel gene will not benefit from inhaled corticosteroids use. Apparently this will save significant time to reach the target of long periods of asthma free symptoms. There is a growing body of opinion that the next breakthrough in asthma management is not going to be a new drug or new class of drugs but rather pharmacogenomics testing to identify targeted therapy in the different phenotypes of asthma.

\section{Inflammatory bowel disease and celiac disease}

Azathioprine (AZA) is frequently used in treatment of Crohn's disease. Absence or low levels of thiopurine methyl transferase (TPMT), an enzyme that is required to metabolize AZA, 6-mercuptopurine (6MP) and thioguanine, could lead to dose dependent side effects or even toxic reactions. It is now required to check the TPMT level and genotype before starting these medicines. Because AZA and 6MP are still the most beneficial available treatments, they may still be used but at low doses depending on TPMT level. Celiac disease is another common disease that might benefit from PM. It has been reported that gut microbiota differs between patients with celiac disease and normal subjects [21]; this finding might pave the road to analyze the microbiota to try to understand whether certain microbiota in the presence or absence of some cell surface molecules may be linked to diagnosis or prognosis or even complete cure of celiac disease.

\section{Acute lymphoblastic leukemia (ALL)}

Currently, approximately $25 \%$ of patients with ALL fail to get complete recovery from ALL [22]. Intravenous gentamycin is frequently used in the patients with ALL to treat the febrile neutropenic patients. Recently, it has been found that some patients have the susceptibility to develop ototoxicity [23, 24]. The most common predisposing mutation is m.1555A which is completely inherited from the maternal side. This mutation was found to cause $50 \%$ of aminoglycoside related hearing loss [25]. In some centers, all the patients who are newly diagnosed with ALL get tested for this mutation and if found to be positive, gentamycin will not be used as one of the treatment options. Moreover, the use of TPMT is not restricted to patients with inflammatory bowel disease as it is also used in leukemic patients and any patient who needs similar immunosuppression.

\section{Childhood diabetes and PM}

Diabetes in children remains one of the areas that is still not well understood. Most of the studies were done in adult population. Pearson et al reported use of sulfonylureas in infants with KCNJ11 mutations, which encode Kir6 ATP sensitive potassium channel [26]. This mutation is thought to be responsible for $50 \%$ of diabetes presentations in infancy. Ninety percent of patients were successfully weaned from insulin to sulfonylurea and the effect was persistent for 1 year at least with good glycemic control and symptoms improvement with a low normal HBA1C.

\section{Pediatric neurology}

PM approach is of particular importance to pediatric neurological diseases. Lysosomal storage disorders (LSDs) are a group of around 40 disorders that affect one in 5,000 babies [27]. LSDs are rare but serious neuro-developmental diseases with well-known genetics bases with fairly understood enzyme deficiencies [6]. There is a possibility of early diagnosis and potential early treatment. Neurodegenerative disorders in children are rare but hold serious consequences. They may lead to neurological disability and severe learning difficulties. The care of such children is time consuming and costly. LSDs are characterized by deficiency or dysfunction of a lysosomal protein or enzyme of any lysosomal compound. This affects the degradation of some macromolecules within the lysosomes leading to gradual build-up of some compounds that would affect the function of the entire cell of many organs including the brain [27]. The accumulation leads to serious effects as it builds up gradually from birth and continues to adulthood leading eventually to neurodegenerative changes that may lead to death in early adulthood. Some scientists believe that understanding and treating neurodegenerative diseases in children could lead to future development of treatment or control of some neurodegenerative disorders in adults, Alzehimer for example. Scarpa et al [6] suggested that LSD could be a good 
example of application of PM for few reasons. First, we understand the genetics of LSDs fairly well and we know that most of the LSDs are due to single gene mutation. They all lead to a reduced enzyme activity. We may be able to prediagnose these if a biomarker is available. Secondly as there are cross-links between different enzyme pathways, discovering one biomarker may lead to adoption of the same principle in treating other conditions. At present various treatments are available targeting slowing the accumulation of the substrate, enzyme replacement therapy (ERT), stabilizing existing enzymes, bone marrow transplant (BMT) and gene therapy. But what we do not know is which patient will need which treatment. Another challenge is discovering an ERT that could cross the blood brain barrier (BBB). If we could find a gene carrying modality to transfer the gene to the defective cell, it would make a huge difference to these disorders and will open the door wide for adoption of such therapy in other genetic diseases.

It is an exciting new era in medicine that will certainly improve care delivered to patients based on individualistic variation. The medical care will be designed to meet the specific patient's needs and to ensure the right and effective treatment is given with less complications and adverse effects from that given treatment.

\section{Conflict of Interest}

The authors declare no conflict of interest.

\section{References}

1. On-line source: Report of Pharmaceutical committee of European commission on the use of -omic technologies in the development of personalised medicine published on 27 March 2013. http://ec.europa.eu/health/files/ committee/70meeting/pharm616.pdf.

2. On-line source: Personalized medicine Coalition: the case for personalized Medicine. Fourth edition. 2014. http:// www.personalizedmedicinecoalition.org/Userfiles/PMCCorporate/file/pmc_the_case_for_personalized_medicine.pdf.

3. On-line source: American Medicine association: personalized Medicine. http://www.ama-assn.org/ama/pub/advocacy/topics/personalized-medicine.page.

4. On-line source: Accenture and National Cancer Institute: Providing real-world personalized medicine. https:// www.accenture.com/us-en/insight-accenture-nci-personalized-medicine-real-world.

5. Elborn JS. The impact of personalised therapies on respiratory medicine. Eur Respir Rev. 2013;22(127):72-74.

6. Scarpa M, Ceci A, Tomanin R, Mincarone P, Begley D. Personalised medicine in paediatrics: individualising treatment in children with rare neurological diseases. EPMA J. 2011;2(2):231-239.

7. Hung SI, Chung WH, Jee SH, Chen WC, Chang YT, Lee WR, Hu SL, et al. Genetic susceptibility to carbamazepine-induced cutaneous adverse drug reactions. Pharmacogenet Genomics. 2006;16(4):297-306.
8. Hetherington S, Hughes AR, Mosteller M, Shortino D, Baker KL, Spreen W, Lai E, et al. Genetic variations in HLA-B region and hypersensitivity reactions to abacavir. Lancet. 2002;359(9312):1121-1122.

9. Kauf TL, Farkouh RA, Earnshaw SR, Watson ME, Maroudas $\mathrm{P}$, Chambers $\mathrm{MG}$. Economic efficiency of genetic screening to inform the use of abacavir sulfate in the treatment of HIV. Pharmacoeconomics. 2010;28(11):10251039.

10. Phillips KA, Veenstra DL, Oren E, Lee JK, Sadee W. Potential role of pharmacogenomics in reducing adverse drug reactions: a systematic review. JAMA. 2001;286(18):2270-2279.

11. Jain KK. Applications of AmpliChip CYP450. Mol Diagn. 2005;9(3):119-127.

12. Carleton B, Poole R, Smith M, Leeder J, Ghannadan R, Ross C, Phillips $\mathrm{M}$, et al. Adverse drug reaction active surveillance: developing a national network in Canada's children's hospitals. Pharmacoepidemiol Drug Saf. 2009;18(8):713-721.

13. Van Goor F, Hadida S, Grootenhuis PD, Burton B, Stack JH, Straley KS, Decker CJ, et al. Correction of the F508del-CFTR protein processing defect in vitro by the investigational drug VX-809. Proc Natl Acad Sci U S A. 2011;108(46):18843-18848.

14. Davies JC, Li H, Yen K, Ahrens R. Ivacaftor in subjects 6 to 11 years of age with cystic fibrosis and the G551DCFTR mutation. JCF. 2012; June(11):S13.

15. Ramsey BW, Davies J, McElvaney NG, Tullis E, Bell SC, Drevinek P, Griese M, et al. A CFTR potentiator in patients with cystic fibrosis and the G551D mutation. N Engl J Med. 2011;365(18):1663-1672.

16. Wainwright CE, Elborn JS, Ramsey BW. LumacaftorIvacaftor in Patients with Cystic Fibrosis Homozygous for Phe508del CFTR. N Engl J Med. 2015;373(18):17831784.

17. Clancy JP, Rowe SM, Accurso FJ, Aitken ML, Amin RS, Ashlock MA, Ballmann M, et al. Results of a phase IIa study of VX-809, an investigational CFTR corrector compound, in subjects with cystic fibrosis homozygous for the F508del-CFTR mutation. Thorax. 2012;67(1):1218.

18. Smith DE. IL-33: a tissue derived cytokine pathway involved in allergic inflammation and asthma. Clin Exp Allergy. 2010;40(2):200-208.

19. Basu K, Palmer CN, Tavendale R, Lipworth BJ, Mukhopadhyay S. Adrenergic beta(2)-receptor genotype predisposes to exacerbations in steroid-treated asthmatic patients taking frequent albuterol or salmeterol. J Allergy Clin Immunol. 2009;124(6):1188-1194 e1183.

20. Kelan G, Tantisira, Lasky-Su J, Harada M, Murphy A, et al. Genomewide Association between GLCCI1 and Response to Glucocorticoid Therapy in Asthma. N Engl J Med. 2011;365:1173-1183.

21. Cheng J, Kalliomaki M, Heilig HG, Palva A, Lahteenoja $\mathrm{H}$, de Vos WM, Salojarvi J, et al. Duodenal microbiota composition and mucosal homeostasis in pediatric celiac disease. BMC Gastroenterol. 2013;13:113.

22. Carroll WL, Bhojwani D, Min DJ, Raetz E, Relling M, 
Davies S, Downing JR, et al. Pediatric acute lymphoblastic leukemia. Hematology Am Soc Hematol Educ Program. 2003:102-131.

23. Qian Y, Guan MX. Interaction of aminoglycosides with human mitochondrial $12 \mathrm{~S}$ rRNA carrying the deafnessassociated mutation. Antimicrob Agents Chemother. 2009;53(11):4612-4618.

24. Muyderman H, Sims NR, Tanaka M, Fuku N, Raghupathi $\mathrm{R}$, Thyagarajan D. The mitochondrial T1095C mutation increases gentamicin-mediated apoptosis. Mitochondri- on. 2012;12(4):465-471.

25. Bitner-Glindzicz M, Rahman S. Ototoxicity caused by aminoglycosides. BMJ. 2007;335(7624):784-785.

26. Pearson ER, Flechtner I, Njolstad PR, Malecki MT, Flanagan SE, Larkin B, Ashcroft FM, et al. Switching from insulin to oral sulfonylureas in patients with diabetes due to Kir6.2 mutations. N Engl J Med. 2006;355(5):467-477.

27. Meikle PJ, Hopwood JJ, Clague AE, Carey WF. Prevalence of lysosomal storage disorders. JAMA. 1999;281(3):249254. 